\title{
$\begin{array}{ll}\text { Research Square } & \text { Preprints are preliminary reports that have not undergone peer review. } \\ \text { They should not be considered conclusive, used to inform clinical practice, }\end{array}$ or referenced by the media as validated information. \\ Preterm birth determinants among newborns at six public hospitals in Northeast Amhara, Ethiopia: unmatched case-control study
}

\section{Mesfin Shiferaw}

Debre Berhan Health Science College

Nigusie Birhan Tebeje

University of Gondar College of Medicine and Health Sciences

Habtamu Sewunet Mekonnen ( $\nabla$ habtsew@ymail.com )

University of Gondar College of Medicine and Health Sciences https://orcid.org/0000-0001-9396-7437

\section{Research}

Keywords: Determinants, preterm birth, newborns, public hospitals, northeast, Ethiopia

Posted Date: March 27th, 2020

DOI: https://doi.org/10.21203/rs.3.rs-19456/v1

License: (a) (i) This work is licensed under a Creative Commons Attribution 4.0 International License. Read Full License 


\section{Abstract}

\section{Background}

Preterm birth is a global public health problem. Worldwide, every year about 15 million newborns are born preterm and about 1.1 million of them die due to complications related to prematurity. More than $81 \%$ of preterm births were in Asia and Sub-Saharan Africa. Nowadays, preterm birth is the first leading cause of under-five mortality and is one of the limiting factors for achieving a reduction in under-five mortality rate. Therefore, the aim of this study was to identify the determinants of preterm birth at public hospitals in the Northeast Amhara, Ethiopia, 2019.

Method

Hospital-based unmatched case-control study was conducted at public hospitals in Northeast Amhara, Ethiopia from March 15 th to April 25 th , 2019. Data were collected using interviewer-administered structured pre-tested tool and document review for cases and controls. Data were entered in to Epi Info version 7.2.2.6 and exported to SPSS version 23 software for data analysis. Bi-variable and multivariable binary logistic regression were used to measure the association between preterm birth and risk factors. Adjusted Odds ratio (AOR) at $95 \%$ confidence interval (Cl) was calculated and $\mathrm{P}$ value $\leq 0.05$ declared statistically significant.

Result

The study identified that ANC follow up less than four visits [AOR 2.267, $\mathrm{Cl}(1.205,4.264)$ ], pregnancyinduced hypertension [AOR 4.453, $\mathrm{Cl}(1.276,15.536)]$, no iron/folic acid supplementation during pregnancy [AOR 4.175, $\mathrm{Cl}(1.632,10.680)]$, anemia during pregnancy [AOR 4.776, $\mathrm{Cl}(1.492,15.285)]$, and birth defect [AOR 6.160, Cl $(I .896,20.013)]$ were significantly associated determinants of preterm birth.

\section{Conclusion}

Antenatal care follow up fewer than four visits, pregnancy induced hypertension, no iron/folic acid supplementation during pregnancy, being anemic during pregnancy, and birth defect were important determinants of preterm birth.

\section{Background}

Preterm birth (PTB), commonly defined as births before 37 completed weeks of gestation or fewer than 259 days since the first day of a woman's last normal menstrual period (LNMP) (1).

The magnitude of PTB shows variation among different parts of the world and is growing through time. Globally, it is estimated that 15 million babies are born too soon each year and the majority of PTBs were in Asia and Sub-Saharan Africa where health systems are weak in access and minimum health services utilization. Additionally, preterm complications account for more than one million neonatal deaths each year(2,3). From 11 countries which have greater than $15 \%$ prevalence of prematurity, all countries are 
found in Sub-Saharan African except Pakistan and Indonesia. Besides, poor countries have a higher prevalence of preterm births than rich countries (4). In Ethiopia, the overall PTB rate is $12 \%$ and the prevalence of PTB in different parts of the country ranges from $4.4-25.9 \%(2,5,6)$

For the first time in history, PTB is the leading cause of death in children younger than 5 years worldwide covering $17 \%$ of under-five and $35 \%$ of neonatal deaths(7-9). Premature infants are particularly vulnerable to complications due to impaired respiration, difficulty in feeding, poor body temperature regulation and high risk of infection. Babies who are born before they are physically ready to face the world encounter greater risks of serious health problems, including cerebral palsy, intellectual impairment, chronic lung disease, and vision and hearing loss $(4,10)$.

Premature babies also face drastically different odds of death depending on where they are born. From those extremely preterm babies ( $<28$ weeks) born in developing countries, greater than $90 \%$ of them die, while greater than $90 \%$ of extremely preterm babies in developed countries survive(4).

Among the 10 countries with the highest numbers of babies dying from PTB complications, India is the first followed by Nigeria. Ethiopia takes the 8th place with 24,400 child deaths per year (9). Different interventions have been implemented to prevent and improve the survival of PTB which demands huge financial capacity imposing a huge economic burden for the family, the community and the nation at large $(4,11)$.

Every Women Every Child initiative set a target for 50\% reduction in PTB deaths by 2025 which needs governments and the global community to take direct action to address deaths due to preterm birth, otherwise, the global total of preterm deaths will not reduce significantly, with around 900,000 premature babies continuing to die every year by 2025(12).

Several risk factors for PTB have been identified across the world. The potential risk factors for PTB includes, but not limited, maternal risk factors like past adverse birth outcomes(PTB, stillbirth, LBW), obstetric complications(PIH, PROM), maternal infections(HIV, malaria, UTI) and no ANC follow up while having $\geq 4$ ANC checkup, IPI 24-36 months, maternal age 20-35 were found to be protective for PTB (1315). However, risk factors for PTB are not always global; risk assessment should be carried out progressively at local, regional, and then global levels(16).

In poorer countries, like Ethiopia, where fewer resources are destined for healthcare, the direct, indirect, immediate and later repercussions of prematurity constitute a relevant public health issue. However, the great majority of scientific evidence accumulated on this subject is generated in developed countries. Besides, the potential risk factors are varied in different locations. Therefore, this study will have its own contribution to avert PTB and its consequences by identifying risk factors.

\section{Method}

\section{Study design and period}


A facility-based unmatched case-control study was conducted at public hospitals in Northeast Amhara, Ethiopia from March 15th to April 25th, 2019.

\section{Study Area}

The study was conducted in Northeast part of Amhara region, Ethiopia. The study was employed in six randomly selected governmental hospitals (Debre Birhan Referral hospital, Tarmaber Primary Hospital, Kemisie General Hospital, Dessie Referral Hospital, Borumeda Hospital, and Woldia general Hospital).

\section{Source Population}

All newborns who were born in public hospitals of Northeast Amhara, Ethiopia.

\section{Study Population}

All newborns who were born in selected public hospitals of Northeast Amhara, during data collection time.

\section{Inclusion And Exclusion Criteria}

\section{Inclusion criteria}

\section{Cases}

All alive singleton preterm newborns (<37 completed weeks of gestation) who was born in study hospitals of northeast Amhara.

\section{Controls}

All alive singleton term newborns (37 completed weeks to 42 weeks) at the same hospitals.

\section{Exclusion Criteria}

- Newborns whose mothers did not remember their LMP.

- Newborns whose mothers had a serious illness or with difficulty of communication during the study period.

\section{Sample Size Determination}

Sample size was determined by using EPI info version 7.2.2.6 by a double-population proportion difference formula. Different variables that have a high contribution for PTB was used and the variable that yields the 
highest sample size was selected. The sample size was calculated considering the following assumptions: $r=3$ (ratio of controls to cases), $80 \%$ power and $95 \%$ confidence level. ANC $<4$ with P Control $=42 \%, P$ Case $=60.7 \%$ was selected. Based on this, the sample size was 323 . By considering a $10 \%$ non-response rate, the final sample size was 356 with 89 cases and 267 controls.

\section{Sampling Technique}

The total sample size was allocated proportionately according to their caseloads for selected hospitals. Cases were preterm newborns (gestational age $<37$ completed weeks) and controls were term newborns (gestational age of $\geq 37$ weeks to 42 weeks). Systematic sampling technique was employed to select controls and cases every fifth intervals and every two intervals respectively. The first participant was selected by lottery method.

\section{Study Variables}

\section{Dependent variable}

Preterm birth (Yes/No)

\section{Independent Variables}

Socioeconomic factors (including paternal age \& education), obstetric history related factors, history of adverse birth outcomes, Obstetric complications, maternal medical conditions, behavioral factors, maternal anthropometrics, and infant characteristics.

\section{Operational Definition}

Preterm birth

Newborns born before 37 completed weeks of gestation

underweight

Mothers with MUAC less than $23 \mathrm{~cm}(17,18)$

Alcohol drinking

$(19,20)$

A unit of alcohol

It is equivalent to $17.7 \mathrm{ml}$ or $14 \mathrm{~g}$ of pure strength alcohol

Binge drinkers

mothers who drink $\geq 3$ drinks at a time/per seat

Drinkers: mothers who use at least one unit of any alcohol during their pregnancy categorized into three levels: 


\section{Data Collection Tools And Procedures}

Data was collected using interviewer-administered, structured pretested questionnaires and record review. The questionnaires include data on socio-demographic characteristics, obstetrics characteristics, infant characteristics, maternal anthropometrics, medical disorders, maternal infections, and behavioral factors. The questionnaires were prepared by reviewing different kinds of literature from related studies and EDHS 2016, (14,15,21-23). Confirmation of Gestational age was taken from documentation and for nonrecorded data, information was taken from mother's report of LNMP. The data was collected by six trained midwifery graduates and supervised by MSc nurses.

MUAC was measured by using flexible non- stretched tape taken at mid-point of left upper arm at relaxed position, without any clothing and with optimal tape tension between acromion process on shoulder blade and tip of olecranon process of ulna. Height was also measured using height Stadiometer with sliding head bar without shoes, standing erect, with shoulder blades, buttocks and heels touching the measuring board, looking straight ahead, shoulders relaxed, arms at sides, legs straight and knees together, feet flat and with heels together. Two height measurements were taken and average was used if readings are within $1 \mathrm{~cm}$ each other and record to the nearest $1 \mathrm{~cm}$.

\section{Data Quality Control}

Data collectors and supervisor were trained for half a day on objective and methods of the study, study tools, how to approach the patient, and take anthropometric measures. In order to assess the validity and reliability of the tool and clarity of the variable, pre-test was done on $5 \%$ of the sample size newborns who were delivered out of the study hospitals two weeks ahead of data collection. During the data collection process, regular monitoring and supervision of the overall activity was done by the supervisors and principal investigator to check for completeness and ensure the quality of data. Besides, data cleaning and coding was carried out before analysis. The measurement tool was prepared in English and translated into local language (Amharic).

\section{Data Processing And Analysis}

The collected data were checked for completeness and entered into EPI info version 7.2.2.6 and exported to SPSS version 23 for analysis. Data were summarized using text, tables, and graphs. Model goodness of fit test was checked by Hosmer-Lemeshow test (P-value $=0.224)$ and fitted. Multicollinearity between independent variables was assessed by using VIF (1.1-1.27) and no problem detected. Binary logistic regression model was used to measure the association between preterm birth and risk factors. Variables that had P-value $\leq 0.2$ on bi-variable regression analysis and had association with preterm birth in 
previous studies were fitted into the multivariable logistic regression model to assess their association with the outcome variable. Adjusted Odds ratio (AOR) with $95 \%$ confidence interval (Cl) was calculated and $\mathrm{P}$ value $\leq 0.05$ declared statistically significant.

\section{Results}

\section{Socio-demographic characteristics of respondents}

In this study, 356 participants were included. From these participants, 89 were preterm newborns (cases) and 267 were term newborns as controls with $100 \%$ response rate. The median age of mothers was $25 \pm 8$ (IQR) years in case group and $26 \pm 7$ (IQR) years in control group with a minimum of 17 and a maximum of 45 years. The paternal median age was $31 \pm 7$ (IQR) years ranges from 20 to 60 years. Regarding religion, 50 (56.2\%) cases and 129 (48.3\%) controls were Orthodox Christian. Majority of the respondents, $69(77.5 \%)$ cases and $220(82.4 \%)$ controls were married and living together. Concerning the educational level of parents, $34(38.2 \%)$ mothers and $27(30.4 \%)$ fathers in the case group had no formal education while $108(44.4 \%)$ mothers and $89(41.1 \%)$ fathers in the control group. About occupation, 46 (51.7\%) cases and $132(49.4 \%)$ controls were housewives. Forty-eight (53.9\%) newborns in case-group and 148 (55.4\%) newborns in control-group were male in sex. (Table 1)

Table 1: Distribution of socio-demographic characteristics among mothers who gave birth at public hospitals of Northeast Amhara, Ethiopia, 2019 


\begin{tabular}{|c|c|c|c|c|}
\hline Variable & Category & Cases (\%) & $\begin{array}{c}\text { Controls } \\
(\%)\end{array}$ & Total \\
\hline \multirow[t]{2}{*}{ Residence } & Rural & $\begin{array}{c}42 \\
(47.2 \%)\end{array}$ & $98(36.7 \%)$ & $\begin{array}{c}140 \\
(39.3 \%)\end{array}$ \\
\hline & Urban & $\begin{array}{c}47 \\
(52.8 \%)\end{array}$ & $\begin{array}{c}169 \\
(63.3 \%)\end{array}$ & $\begin{array}{c}216 \\
(60.7 \%)\end{array}$ \\
\hline \multirow[t]{3}{*}{ Age of mother } & $<20$ & $6(6.7 \%)$ & $10(3.7 \%)$ & $16(4.5 \%)$ \\
\hline & $20-34$ & $\begin{array}{c}68 \\
(76.4 \%)\end{array}$ & $\begin{array}{c}226 \\
(84.6 \%)\end{array}$ & $\begin{array}{c}294 \\
(82.6 \%)\end{array}$ \\
\hline & $\geq 35$ & $15(16.9 \%$ & $31(11.6 \%)$ & $46(12.9 \%)$ \\
\hline \multirow[t]{5}{*}{ Educational status } & $\begin{array}{l}\text { Not able to read and } \\
\text { write }\end{array}$ & $\begin{array}{c}20 \\
(22.5 \%)\end{array}$ & $58(21.7 \%)$ & $78(21.9 \%)$ \\
\hline & Able to read and write & $\begin{array}{c}14 \\
(15.7 \%)\end{array}$ & $50(18.7 \%)$ & $64(18.0 \%)$ \\
\hline & Primary education & $\begin{array}{c}19 \\
(21.3 \%)\end{array}$ & $58(21.7 \%)$ & 77 (21.6\%) \\
\hline & Secondary education & $\begin{array}{c}23 \\
(25.8 \%)\end{array}$ & $60(22.5 \%)$ & $83(23.3 \%)$ \\
\hline & Higher education & $\begin{array}{c}13 \\
(14.6 \%)\end{array}$ & $41(15.4 \%)$ & $54(15.2 \%)$ \\
\hline \multirow[t]{3}{*}{ Marital status } & Married & $\begin{array}{c}69 \\
(77.5 \%)\end{array}$ & $\begin{array}{c}220 \\
(82.4 \%)\end{array}$ & $\begin{array}{c}289 \\
(81.2 \%)\end{array}$ \\
\hline & Separated & $\begin{array}{c}16 \\
(18.0 \%)\end{array}$ & $30(11.2 \%)$ & $46(12.9 \%)$ \\
\hline & Single/Divorced/Widowed & $4(4.5 \%)$ & $17(6.4 \%)$ & $21(5.9 \%)$ \\
\hline \multirow[t]{4}{*}{ Religion } & Orthodox & $\begin{array}{c}50 \\
(56.2 \%)\end{array}$ & $\begin{array}{c}129 \\
(48.3 \%)\end{array}$ & $\begin{array}{c}179 \\
(50.3 \%)\end{array}$ \\
\hline & Muslim & $\begin{array}{c}33 \\
(37.1 \%)\end{array}$ & $\begin{array}{c}120 \\
(44.9 \%)\end{array}$ & $\begin{array}{c}153 \\
(43.0 \%)\end{array}$ \\
\hline & Protestant & $5(5.6 \%)$ & $13(4.9 \%)$ & $18(5.1 \%)$ \\
\hline & Catholic & $1(1.1 \%)$ & $5(1.9 \%)$ & $6(1.7 \%)$ \\
\hline \multirow[t]{4}{*}{ Occupational status } & Housewife & $\begin{array}{c}46 \\
(51.7 \%)\end{array}$ & $\begin{array}{c}132 \\
(49.4 \%)\end{array}$ & $\begin{array}{c}178 \\
(50.0 \%)\end{array}$ \\
\hline & Farmer & $\begin{array}{c}10 \\
(11.2 \%)\end{array}$ & $30(11.2 \%)$ & $40(11.2 \%)$ \\
\hline & Merchant & $\begin{array}{c}14 \\
(15.7 \%)\end{array}$ & $40(15.0 \%)$ & $54(15.2 \%)$ \\
\hline & $\begin{array}{c}\text { Government/NOGs } \\
\text { employee }\end{array}$ & $\begin{array}{c}19 \\
(21.3 \%)\end{array}$ & $65(24.3 \%)$ & $84(23.6 \%)$ \\
\hline \multirow[t]{5}{*}{$\begin{array}{l}\text { Educational status of } \\
\text { father }\end{array}$} & $\begin{array}{l}\text { Not able to read and } \\
\text { write }\end{array}$ & $\begin{array}{c}11 \\
(12.4 \%)\end{array}$ & $45(16.9 \%)$ & $56(15.7 \%)$ \\
\hline & Able to read and write & $\begin{array}{c}16 \\
(18.0 \%)\end{array}$ & $54(20.2 \%)$ & $70(19.7 \%)$ \\
\hline & Primary education & $\begin{array}{c}21 \\
(23.6 \%)\end{array}$ & $49(18.4 \%)$ & $70(19.7 \%)$ \\
\hline & Secondary education & $\begin{array}{c}25 \\
(28.1 \%)\end{array}$ & $62(23.2 \%)$ & $87(24.4 \%)$ \\
\hline & Higher education & $\begin{array}{c}16 \\
(18.0 \%)\end{array}$ & $57(21.3 \%)$ & $73(20.5 \%)$ \\
\hline \multirow[t]{3}{*}{ Age of father } & $<25$ & $5(5.6 \%)$ & $10(3.7 \%)$ & $15(4.2 \%)$ \\
\hline & $25-29$ & $\begin{array}{c}30 \\
(33.7 \%)\end{array}$ & $63(23.6 \%)$ & $93(26.1 \%)$ \\
\hline & $30-34$ & $\begin{array}{c}25 \\
(28.1 \%)\end{array}$ & $95(35.6 \%)$ & $\begin{array}{c}120 \\
(33.7 \%)\end{array}$ \\
\hline
\end{tabular}




\begin{tabular}{ccccc} 
& $35-39$ & 16 & $49(18.4 \%)$ & $65(18.3 \%)$ \\
& & $(18.0 \%)$ & & \\
Income (monthly) & 13 & $50(18.7 \%)$ & $63(17.7 \%)$ \\
& & $(14.6 \%)$ & & \\
& & 52 & 130 & 182 \\
Infant sex & & $(58.4 \%)$ & $(48.7 \%)$ & $(51.1 \%)$ \\
& & 37 & 137 & 174 \\
& & $(41.6 \%)$ & $(51.3 \%)$ & $(48.9 \%)$ \\
& & 48 & 148 & 196 \\
& Male & $(53.9 \%)$ & $(55.4 \%)$ & $(55.1 \%)$ \\
\hline & Female & 41 & 119 & $1 .(44.9 \%)$
\end{tabular}

\section{Maternal Obstetric Related Factors}

In this study, 66 (74.2\%) cases and 113 (42.3\%) controls had fewer than four ANC visits. Among these, 25 (28.1\%) cases and 13 (4.9\%) controls had no ANC follow up. Regarding Iron/folic acid supplementation, 44 $(49.4 \%)$ cases and 45 (16.9\%) controls had used no Iron/folic acid. About 13 (14.6\%) cases and 7 (2.6\%) controls had experienced anemia during pregnancy. The majority, $66(74.2 \%)$ newborns in case-group and 217 (81.3\%) newborns in control-group were born through spontaneous vaginal delivery. Concerning, past adverse birth outcome, $8(25 \%)$ cases and 18 (17.3\%) controls had one or more past adverse birth outcomes. Past adverse birth outcomes encountered were LBW 5 (15.6\%), Stillbirth 3 (9.4\%), and Abortion 3 (9.4\%) among cases and 6 (5.8\%), 9 (8.7\%), and 7 (6.7\%) among controls respectively. (Table 2) 
Table 2

Maternal obstetric related factors among mothers who gave birth at public hospitals of Northeast Amhara, Ethiopia, 2019

\begin{tabular}{|c|c|c|c|c|}
\hline Variable & Category & Cases (\%) & Controls (\%) & Total \\
\hline \multirow[t]{3}{*}{ ANC Follow up } & No & $25(28.1 \%)$ & $13(4.9 \%)$ & $38(10.7 \%)$ \\
\hline & $<4$ & $41(46.1 \%)$ & $100(37.5 \%)$ & $141(39.6 \%)$ \\
\hline & $\geq 4$ & $23(25.8 \%)$ & $154(57.7 \%)$ & $177(49.7 \%)$ \\
\hline \multirow[t]{3}{*}{ Time of ANC initiation } & First trimester & $23(35.9 \%)$ & $97(38.2 \%)$ & $120(37.7 \%)$ \\
\hline & Second trimester & $40(62.5 \%)$ & $150(59.1 \%)$ & $190(59.7 \%)$ \\
\hline & Third trimester & $1(1.6 \%)$ & $7(2.8 \%)$ & $8(2.5 \%)$ \\
\hline \multirow[t]{4}{*}{ Birth interval } & No previous birth & $57(64.0 \%)$ & $163(61.0 \%)$ & $220(61.8 \%)$ \\
\hline & $<24$ months & $5(5.6 \%)$ & $19(7.1 \%)$ & $24(6.7 \%)$ \\
\hline & $24-36$ months & $20(22.5 \%)$ & $68(25.5 \%)$ & $88(24.7 \%)$ \\
\hline & $\geq 37$ months & $7(7.9 \%)$ & $17(6.4 \%)$ & $24(6.7 \%)$ \\
\hline \multirow[t]{2}{*}{ Parity } & Primipara & $57(64.0 \%)$ & $163(61.0 \%)$ & $220(61.8 \%)$ \\
\hline & Multipara & $32(36.0 \%)$ & $104(39.0 \%)$ & $136(38.2 \%)$ \\
\hline \multirow[t]{3}{*}{ Iron/folic acid supplementation } & No & $44(49.4 \%)$ & $45(16.9 \%)$ & $89(25.0 \%)$ \\
\hline & $30-60$ tabs & $36(40.4 \%)$ & $144(53.9 \%)$ & $180(50.6 \%)$ \\
\hline & $>60$ tabs & $9(10.1 \%)$ & $78(29.2 \%)$ & $87(24.4 \%)$ \\
\hline \multirow[t]{2}{*}{ Anemia } & No & 76 (85.4\%) & 260 (97.4\%) & 336 (94.4\%) \\
\hline & Yes & $13(14.6 \%)$ & $7(2.6 \%)$ & $20(5.6 \%)$ \\
\hline \multirow{2}{*}{$\begin{array}{l}\text { Hemoglobin } \\
\text { Level }\end{array}$} & $<11 \mathrm{~g} / \mathrm{dl}$ & $25(28.1 \%)$ & $35(13.1 \%)$ & $60(16.9 \%)$ \\
\hline & $\geq 11 \mathrm{~g} / \mathrm{dl}$ & $64(71.9 \%)$ & $232(86.9 \%$ & $296(83.1 \%)$ \\
\hline \multirow[t]{3}{*}{ Mode of delivery } & Spontaneous & $66(74.2 \%)$ & $217(81.3 \%)$ & $283(79.5 \%)$ \\
\hline & Induction & $18(20.2 \%)$ & $16(6.0 \%)$ & $34(9.6 \%)$ \\
\hline & C-section & $5(5.6 \%)$ & $34(12.7 \%)$ & $39(11.0 \%)$ \\
\hline \multirow[t]{2}{*}{ Pregnancy status } & Unplanned & $22(24.7 \%)$ & $33(12.4 \%)$ & $55(15.4 \%)$ \\
\hline & Planned & 67 (75.3\%) & $234(87.6 \%)$ & $301(84.6 \%)$ \\
\hline \multirow[t]{2}{*}{ Maternal Rh factor } & Positive & $59(66.3 \%)$ & $187(70.0 \%)$ & $246(69.1 \%)$ \\
\hline & Negative & 25 (28.1\%) & 72 (27.0\%) & $97(27.2 \%)$ \\
\hline
\end{tabular}




\begin{tabular}{|lllll|}
\hline Variable & Category & Cases (\%) & Controls (\%) & Total \\
\hline Maternal height & Unknown & $5(5.6 \%)$ & $8(3.0 \%)$ & $13(3.7 \%)$ \\
\hline Maternal MUAC & $<150$ & $20(22.5 \%)$ & $35(13.1 \%)$ & $55(15.4 \%)$ \\
\cline { 2 - 5 } & $\geq 150$ & $69(77.5 \%)$ & $232(86.9 \%)$ & $301(84.6 \%)$ \\
\hline History of adverse birth outcome & $\mathbf{2}$ & $44(49.4 \%)$ & $88(33.0 \%)$ & $132(37.1 \%)$ \\
\cline { 2 - 5 } & No & $45(50.6 \%)$ & $179(67.0 \%)$ & $224(62.9 \%)$ \\
\cline { 2 - 5 } & Yes & $24(75.0 \%$ & $86(82.7 \%)$ & $110(80.9 \%)$ \\
\hline $\begin{array}{l}\text { Type of adverse } \\
\text { birth outcome }\end{array}$ & LBW & $5(25.0 \%)$ & $18(17.3 \%)$ & $26(19.1 \%)$ \\
\cline { 2 - 5 } & Stillbirth & $3(9.4 \%)$ & $9(8.7 \%)$ & $12(8.8 \%)$ \\
\cline { 2 - 5 } & Abortion & $3(9.4 \%)$ & $7(6.7 \%)$ & $10(7.4 \%)$ \\
\hline
\end{tabular}

Table 3

Maternal infection and chronic disease related factors among mothers who gave birth at public hospitals of Northeast Amhara, Ethiopia, 2019

\begin{tabular}{|lllll|}
\hline Variable & Category & Cases (\%) & Controls (\%) & Total \\
\hline \multirow{2}{*}{ Chronic medical illness } & No & $83(93.3 \%)$ & $253(94.8 \%)$ & $336(94.4 \%)$ \\
\cline { 2 - 5 } & Yes & $6(6.7 \%)$ & $14(5.2 \%)$ & $20(5.6 \%)$ \\
\hline \multirow{2}{*}{ Type of medical illness } & HPN & $3(3.4 \%)$ & $4(1.5 \%)$ & $7(2.0 \%)$ \\
\cline { 2 - 5 } & DM/KD/LD?TD/ & $4(4.49 \%)$ & $10(3.74 \%)$ & $14(3.93 \%)$ \\
\hline Maternal infection & UTI & $10(11.2 \%)$ & $24(9.0 \%)$ & $34(9.6 \%)$ \\
\cline { 2 - 5 } & Malaria & $1(1.1 \%)$ & $4(1.5 \%)$ & $5(1.4 \%)$ \\
\cline { 2 - 5 } & TB & $1(1.1 \%)$ & $5(1.9 \%)$ & $6(1.7 \%)$ \\
\cline { 2 - 5 } & HIV/AIDS & $0(\% 0)$ & $8(3.0 \%)$ & $8(2.2 \%)$ \\
\hline Key: DM = diabetes mellitus, KD = kidney disease, LD = liver disease, TD = thyroid disorder \\
\hline
\end{tabular}


Table 4

Behavioral factors among mothers who gave birth at public hospitals of Northeast Amhara, Ethiopia, 2019

\begin{tabular}{|c|c|c|c|c|}
\hline Variable & Category & $\begin{array}{l}\text { Cases } \\
(\%)\end{array}$ & $\begin{array}{l}\text { Controls } \\
(\%)\end{array}$ & Total \\
\hline \multirow[t]{2}{*}{ Alcohol drinking during pregnancy } & No & $\begin{array}{l}62 \\
(69.7 \%)\end{array}$ & $\begin{array}{l}199 \\
(74.5 \%)\end{array}$ & $\begin{array}{l}261 \\
(73.3 \%)\end{array}$ \\
\hline & Yes & $\begin{array}{l}27 \\
(30.3 \%)\end{array}$ & $68(25.5 \%)$ & $95(26.7 \%)$ \\
\hline \multirow[t]{4}{*}{ Type of alcohol drunk } & Tella & $\begin{array}{l}19 \\
(21.3 \%)\end{array}$ & $56(21.0 \%$ & $75(21.1 \%)$ \\
\hline & Areke & $6(6.7 \%)$ & $7(2.6 \%)$ & $13(3.7 \%)$ \\
\hline & Beer & $8(9.0 \%)$ & $24(9.0 \%)$ & $32(9.0 \%)$ \\
\hline & Wine/Tej & $7(7.9 \%)$ & $7(2.6 \%)$ & $14(3.9 \%)$ \\
\hline \multirow[t]{3}{*}{ Frequency of drinking } & Daily/Weekly & $9(33.3 \%)$ & $13(19.1)$ & $22(23.2 \%)$ \\
\hline & Fortnightly/Monthly & $7(25.9 \%)$ & $13(19.1 \%)$ & $20(21.0 \%)$ \\
\hline & Occasionally & $\begin{array}{l}11 \\
(40.7 \%)\end{array}$ & $42(61.8 \%)$ & $53(55.8 \%)$ \\
\hline \multirow[t]{3}{*}{$\begin{array}{l}\text { Unit of alcohol per week (recent } \\
\text { month) }\end{array}$} & No alcohol & $\begin{array}{l}65 \\
(73.0 \%)\end{array}$ & $\begin{array}{l}223 \\
(83.5 \%)\end{array}$ & $\begin{array}{l}288 \\
(80.9 \%)\end{array}$ \\
\hline & $1-3$ unit & $\begin{array}{l}21 \\
(23.6 \%)\end{array}$ & $40(15.0 \%)$ & $61(17.1 \%)$ \\
\hline & $\geq 4$ unit & $3(3.4 \%)$ & $4(1.5 \%)$ & $7(2.0 \%)$ \\
\hline \multirow[t]{2}{*}{ Khat chewing during pregnancy } & No & $\begin{array}{l}84 \\
(94.4 \%)\end{array}$ & $\begin{array}{l}248 \\
(92.9 \%)\end{array}$ & $\begin{array}{l}332 \\
(93.3 \%)\end{array}$ \\
\hline & Yes & $5(5.6 \%)$ & $19(7.1 \%)$ & $24(6.7 \%)$ \\
\hline \multirow[t]{4}{*}{ Frequency of khat chewing } & Daily/Weekly & $1(20 \%)$ & $4(21.1 \%)$ & $5(20.8 \%)$ \\
\hline & Fortnightly & $0(0 \%)$ & $5(26.3 \%)$ & $5(20.8 \%)$ \\
\hline & Monthly & $0(0 \%)$ & $1(5.3 \%)$ & $1(4.2 \%)$ \\
\hline & Occasionally & $4(80 \%)$ & $9(47.4 \%)$ & $13(54.2 \%)$ \\
\hline \multirow[t]{2}{*}{ Passive Smoking } & No & $\begin{array}{l}87 \\
(97.8 \%)\end{array}$ & $\begin{array}{l}261 \\
(97.8 \%)\end{array}$ & $\begin{array}{l}348 \\
(97.8 \%)\end{array}$ \\
\hline & Yes & $2(2.2 \%)$ & $6(2.2 \%)$ & $8(2.2 \%)$ \\
\hline Any medication during pregnancy & No & $\begin{array}{l}74 \\
(83.1 \%)\end{array}$ & $\begin{array}{l}227 \\
(85.0 \%)\end{array}$ & $\begin{array}{l}301 \\
(84.6 \%)\end{array}$ \\
\hline
\end{tabular}




\begin{tabular}{|lllll|}
\hline Variable & Category & $\begin{array}{l}\text { Cases } \\
(\%)\end{array}$ & $\begin{array}{l}\text { Controls } \\
(\%)\end{array}$ & Total \\
\hline Yes & $\begin{array}{l}15 \\
(16.9 \%)\end{array}$ & $40(15.0 \%)$ & $55(15.4 \%)$ \\
\hline Frequency of medication use & Daily & $\begin{array}{l}12 \\
(80.0 \%)\end{array}$ & $34(85.0 \%)$ & $46(83.6 \%)$ \\
\cline { 2 - 5 } & Weekly & $0(0 \%)$ & $1(2.5 \%)$ & $1(1.8 \%)$ \\
\hline & Occasionally & $3(20.0 \%)$ & $5(12.5 \%)$ & $8(14.5 \%)$ \\
\hline
\end{tabular}


Table 5

Bi-variable and Multi-variable analysis of determinants associated with preterm birth among mothers who gave birth at public hospitals of Northeast Amhara, Ethiopia, 2019

\begin{tabular}{|c|c|c|c|c|c|}
\hline Variables & Category & $\begin{array}{l}\text { Cases } \\
(\%)\end{array}$ & $\begin{array}{l}\text { Controls } \\
\text { (\%) }\end{array}$ & $\operatorname{COR}(95 \% \mathrm{Cl})$ & AOR (95\% Cl) \\
\hline \multirow[t]{2}{*}{ Residence } & Rural & $\begin{array}{l}42 \\
(47.2 \%)\end{array}$ & $\begin{array}{l}98 \\
(36.7 \%)\end{array}$ & $\begin{array}{l}1.541(0.949 \\
2.503)\end{array}$ & $0.998(0.521,1.912)$ \\
\hline & Urban & $\begin{array}{l}47 \\
(52.8 \%)\end{array}$ & $\begin{array}{l}169 \\
(63.3 \%)\end{array}$ & 1 & 1 \\
\hline \multirow[t]{2}{*}{$\begin{array}{l}\text { Pregnancy } \\
\text { status }\end{array}$} & Unplanned & $\begin{array}{l}22 \\
(24.7 \%)\end{array}$ & $\begin{array}{l}33 \\
(12.4 \%)\end{array}$ & $\begin{array}{l}2.328(1.273 \\
4.259)\end{array}$ & $0.995(0.442,2.242)$ \\
\hline & Planned & $\begin{array}{l}67 \\
(75.3 \%)\end{array}$ & $\begin{array}{l}234 \\
(87.6 \%)\end{array}$ & 1 & 1 \\
\hline \multirow[t]{2}{*}{ ANC follow up } & $\begin{array}{l}<4 \text { ANC } \\
\text { visit }\end{array}$ & $\begin{array}{l}66 \\
(74.2 \%)\end{array}$ & $\begin{array}{l}113 \\
(42.3 \%)\end{array}$ & $\begin{array}{l}3.911(2.295 \\
6.665)\end{array}$ & $2.267(1.205,4.264)^{\star \star}$ \\
\hline & $\begin{array}{l}\geq 4 \text { ANC } \\
\text { visit }\end{array}$ & $\begin{array}{l}23 \\
(25.8 \%)\end{array}$ & $\begin{array}{l}154 \\
(57.7 \%)\end{array}$ & 1 & 1 \\
\hline \multirow[t]{3}{*}{$\begin{array}{l}\text { Age of mother in } \\
\text { years }\end{array}$} & $<19$ & $\begin{array}{l}6 \\
(6.7 \%)\end{array}$ & $\begin{array}{l}10 \\
(3.7 \%)\end{array}$ & $1.240(0.379,4.056)$ & $1.326(0.290,6.060)$ \\
\hline & $20-34$ & $\begin{array}{l}68 \\
(76.4 \%)\end{array}$ & $\begin{array}{l}226 \\
(84.6 \%)\end{array}$ & $0.622(0.317,1.219)$ & $1.009(0.419,2.431)$ \\
\hline & $\geq 35$ & $\begin{array}{l}15 \\
(16.9 \%)\end{array}$ & $\begin{array}{l}31 \\
(11.6 \%)\end{array}$ & 1 & 1 \\
\hline \multirow[t]{2}{*}{$\mathrm{PIH}$} & No & $\begin{array}{l}75 \\
(84.3 \%)\end{array}$ & $\begin{array}{l}261 \\
(97.8 \%)\end{array}$ & 1 & 1 \\
\hline & Yes & $\begin{array}{l}14 \\
(15.7 \%)\end{array}$ & $6(2.2 \%)$ & $\begin{array}{l}8.120(3.016 \\
21.858)\end{array}$ & $4.453(1.276,15.536)^{\star \star}$ \\
\hline \multirow[t]{2}{*}{ PROM } & No & $\begin{array}{l}77 \\
(86.5 \%)\end{array}$ & $\begin{array}{l}259 \\
(97 \%)\end{array}$ & 1 & 1 \\
\hline & Yes & $\begin{array}{l}12 \\
(13.5 \%)\end{array}$ & $8(3.0 \%)$ & $\begin{array}{l}5.045(1.991 \\
12.789)\end{array}$ & $3.159(0.938,10.638)$ \\
\hline \multirow[t]{2}{*}{$\begin{array}{l}\text { Congenital } \\
\text { malformation }\end{array}$} & No & $\begin{array}{l}74 \\
(83.1 \%)\end{array}$ & $\begin{array}{l}262 \\
(98.1 \%)\end{array}$ & 1 & 1 \\
\hline & Yes & $\begin{array}{l}15 \\
)^{(16.9 \%}\end{array}$ & $5(1.9 \%)$ & $\begin{array}{l}10.622(3.737 \\
30.186)\end{array}$ & $6.160(1.896,20.013)^{\star}$ \\
\hline
\end{tabular}

Key: $\mathrm{COR}=$ crude odds ratio, $\mathrm{AOR}=$ adjusted odds ratio, $\mathrm{Cl}=$ confidence interval, $*=\mathrm{P}$-value $<0.01,{ }^{* *}=\mathrm{p}$ value $\leq 0.05$ 


\begin{tabular}{|c|c|c|c|c|c|}
\hline Variables & Category & $\begin{array}{l}\text { Cases } \\
(\%)\end{array}$ & $\begin{array}{l}\text { Controls } \\
(\%)\end{array}$ & COR (95\% Cl) & AOR (95\% Cl) \\
\hline \multirow[t]{3}{*}{$\begin{array}{l}\text { Iron/folic acid } \\
\text { supplementation }\end{array}$} & $\begin{array}{l}\text { No iron } \\
\text { use }\end{array}$ & $\begin{array}{l}44 \\
(49.4 \%)\end{array}$ & $\begin{array}{l}45 \\
(16.9 \%)\end{array}$ & 8.474(3.78718.962) & $4.175(1.632,10.680)^{\star}$ \\
\hline & $\begin{array}{l}30-60 \\
\text { tabs }\end{array}$ & $\begin{array}{l}36 \\
(40.4 \%)\end{array}$ & $\begin{array}{l}144 \\
(53.9 \%)\end{array}$ & $2.167(0.9934 .730)$ & $1.838(0.765,4.418)$ \\
\hline & $\geq 60$ tabs & $\begin{array}{l}9 \\
(10.1 \%)\end{array}$ & $\begin{array}{l}78 \\
(29.2 \%)\end{array}$ & 1 & 1 \\
\hline \multirow{2}{*}{$\begin{array}{l}\text { Anemia during } \\
\text { current } \\
\text { pregnancy }\end{array}$} & No & $\begin{array}{l}76 \\
(85.4 \%)\end{array}$ & $\begin{array}{l}260 \\
(97.4 \%)\end{array}$ & 1 & 1 \\
\hline & Yes & $\begin{array}{l}13 \\
(14.6 \%)\end{array}$ & $7(2.6 \%)$ & $\begin{array}{l}6.353(2.448 \\
16.489)\end{array}$ & $\begin{array}{l}4.776(1.492 \\
15.285)^{\star}\end{array}$ \\
\hline \multirow[t]{2}{*}{$\begin{array}{l}\text { Hemoglobin } \\
\text { level }\end{array}$} & $<11 \mathrm{mg} / \mathrm{dl}$ & $\begin{array}{l}25 \\
(28.1 \%)\end{array}$ & $\begin{array}{l}35 \\
(13.1 \%)\end{array}$ & $\begin{array}{l}2.589(1.445 \\
4.639)\end{array}$ & $1.880(0.925,3.821)$ \\
\hline & $\geq 11 \mathrm{mg} / \mathrm{dl}$ & $\begin{array}{l}64 \\
(71.9 \%)\end{array}$ & $\begin{array}{l}232 \\
(86.9 \%\end{array}$ & 1 & 1 \\
\hline \multirow[t]{2}{*}{$\begin{array}{l}\text { Height of } \\
\text { Mothers }\end{array}$} & $<150 \mathrm{~cm}$ & $\begin{array}{l}20 \\
(22.5 \%)\end{array}$ & $\begin{array}{l}35 \\
(13.1 \%)\end{array}$ & $\begin{array}{l}1.921(1.042 \\
3.542)\end{array}$ & $1.112(0.513,2.411)$ \\
\hline & $\geq 150 \mathrm{~cm}$ & $\begin{array}{l}69 \\
(77.5 \%)\end{array}$ & $\begin{array}{l}232 \\
(86.9 \%)\end{array}$ & 1 & 1 \\
\hline \multirow[t]{2}{*}{ MUAC } & $<23 \mathrm{~cm}$ & $\begin{array}{l}44 \\
(49.4 \%)\end{array}$ & $\begin{array}{l}88 \\
(33.0 \%)\end{array}$ & $\begin{array}{l}1.989(1.221 \\
3.239)\end{array}$ & $1.481(0.812,2.699)$ \\
\hline & $\geq 23 \mathrm{~cm}$ & $\begin{array}{l}45 \\
(50.6 \%)\end{array}$ & $\begin{array}{l}179 \\
(67.0 \%)\end{array}$ & 1 & 1 \\
\hline \multirow[t]{2}{*}{$\begin{array}{l}\text { Income } \\
\text { (monthly) }\end{array}$} & $\begin{array}{l}<3000 \\
\text { EBR }\end{array}$ & $\begin{array}{l}52 \\
(58.4 \%)\end{array}$ & $\begin{array}{l}130 \\
(48.7 \%)\end{array}$ & $\begin{array}{l}1.481(0.912 \\
2.406)\end{array}$ & $1.388(0.738,2.608)$ \\
\hline & $\begin{array}{l}\geq 3000 \\
\text { EBR }\end{array}$ & $\begin{array}{l}37 \\
(41.6 \%)\end{array}$ & $\begin{array}{l}137 \\
(51.3 \%)\end{array}$ & 1 & 1 \\
\hline
\end{tabular}

\section{Fetal Related Factors}

The mean gestational age among PTBs and term births were $34 \pm 1.94$ weeks and $39.02 \pm 1.27$ weeks, respectively. In the case group, 17 (19.1\%) newborns were small for gestational age whereas 12 (4.5\%) newborns in control group were low birth weight. Concerning fetal distress, 5 (5.6\%) cases and $15(5.6 \%)$ controls had fetal distress. On the other hand, $15(16.9 \%)$ preterm newborns and $5(1.9 \%)$ term newborns had a visible congenital/birth defect. 


\section{Current Obstetric Complications}

During current pregnancy, about 23 (25.8\%) cases and 27 (10.1\%) controls had one or more of pregnancyrelated complications. Pregnancy-related complications occurred among cases were APH 3 (3.4\%), Preeclampsia 2 (2.2\%), PROM 12 (13.5\%), PIH 14 (15.7\%), and Hyperemesis gravidarum 4 (4.5\%) whereas 1 (0.4\%), $3(1.1 \%), 8(3.0 \%), 6(2.2 \%)$, and $11(4.1 \%)$ controls had the same obstetric complications in the same order.

\section{Maternal Infection And Chronic Disease Related Factors}

About 6 (6.7\%) cases and 14 (5.2\%) controls had a chronic medical illness. Chronic hypertension was observed in $3(3.4 \%)$ cases and 4 (1.5\%) controls. Concerning maternal infections, UTI among $10(11.2 \%)$ cases and 24 (9\%) controls, and HIV/AIDS 8 (3.0\%) among controls were found. Table-3

\section{Behavioral And Lifestyle Related Factors}

Twenty-seven (30.3\%) cases and 68 (25.5\%) controls were drunk alcohol. The alcoholic beverage drunk comprised of "Tella", "Tej", "Areke", Beer, and Wine. The majority, 19 (21.3\%) cases and $56(21.0 \%)$ controls drunk "Tella". Regarding Khat chewing during pregnancy, 5 (5.6\%) cases and 19 (7.1\%) controls were chewed khat. About $15(16.9 \%)$ cases and $40(15.0 \%)$ of their counterparts were used medication other than minerals, vitamins, and iron/folic acid prescribed or from the counter. (Table- 4 )

\section{Determinants Associated With Preterm Birth}

In bi-variable logistic regression analysis at $95 \%$ level of confidence, ANC follow up, pregnancy status, PIH, PROM, Congenital malformation, Iron/folic acid supplementation, Anemia and hemoglobin level during current pregnancy, Height of mothers, and MUAC were statistically significant with PTB. In multivariable logistic regression analysis at P-value $\leq 0.05$, ANC follow up, $\mathrm{PIH}$, Congenital malformation, Iron/folic acid consumption, and Anemia during current pregnancy remained significantly associated determinants of PTB.

The chance of being preterm among newborns whose mothers had had fewer than four ANC follow up was about 2 times more than newborns whose mothers had had four or more ANC follow up [AOR 2.267, $\mathrm{Cl}(1.205,4.264)]$. Newborns of mothers with PIH were four and half folds higher to be preterm [AOR 4.453 $\mathrm{Cl}(1.276,15.536)]$ compared to newborns born from mothers with no PIH. Similarly, newborns whose mothers had not used iron/folic acid supplementation during current pregnancy were 4 times increased the risk to be born preterm [AOR 4.175, $(1.632,10.680)]$ compared to their counterparts. The odds of being preterm in newborns with a birth defect were 6 times higher [AOR 6.160, $\mathrm{Cl}(1.896,20.013)$ ] compared to newborns without a birth defect. Additionally, those newborns that born from mothers who had had 
anemia during current pregnancy were about 5 times higher to be born preterm [AOR $4.776(1.492,15.285)$ ] than newborns whose mothers had no anemia. (Table-5)

\section{Discussion}

This study was intended to identify determinants for PTB in order to tackle the burden of the disease and its associated problems. The study has tried to look at the determinants of PTB by incorporating numerous risk factors as possible.

This study revealed that newborns whose mothers followed ANC less than four times were about two times more likely to be preterm as compared to their counterparts. This finding is consistent with facilitybased case-control studies done in Ethiopia; Jimma (AOR: 4.8), and Central Tigray (AOR: 2.15), NorthEastern Tanzania (AOR 3.25), and Nigeria (AOR 6.13). This might be due to that lack of/ inadequate ANC follow up decreases the chance of identifying risks of PTB and providing appropriate interventions for its prevention(14,21).

In this study, current PIH showed statically significant association with PTB. PIH increased the odds of PTB by four and half folds in newborns of mothers having PIH than newborns of mothers without PIH. Similarly, significant associations have been reported from studies done in Ethiopia, Gondar (AOR = 5.36), Jimma (AOR 6), and Central Tigray (AOR 3.24), and other studies from Kenya (7.81), and Iran (AOR 7.30). This might be due to hypertension increases the resistance of blood to uterine vessels reducing the uteroplacental blood flow that may affect the exchange of nutrients and oxygen between mother and fetus which in turn results intrauterine growth restriction that causes PTB. Additionally, complications of PIH may cause vascular damage to the placenta, which induces the oxytocin receptors, leads to preterm labor and delivery (14,24-26). Even though this may not be causal in nature, $\mathrm{PIH}$ is a common reason for indicated preterm deliveries and this may explain its association with PTB.

This study also showed that anemia during current pregnancy were another independent determinant for PTB. Newborns whose mothers had anemia during current pregnancy were at increased risk to be born preterm compared to those newborns of mothers without anemia. This finding is in line with the studies conducted in Ethiopia, Jimma (AOR 2.7), Systematic review and meta-analysis in low- and middle-income countries (RR: 1.63) and Korea (AOR 1.53). This might be due to that anemia and iron deficiency by causing hypoxia and maternal infections induce maternal and fetal stress which leads to the formation of corticotrophin-releasing hormone. Excess corticotrophin-releasing hormone is the major risk factor for preterm labor, PIH, PROM and inhibits fetal growth by stimulating cortisol production. Another possible explanation is that iron deficiency may cause oxidative damage to the erythrocytes and the feto-placental unit affecting placental function $(27,28)$.

The present study revealed that the chance of being preterm among neonates having birth defect was about six times more significant compared to those neonates without any visible birth defect. This finding agrees with the studies done in Tigray, Ethiopia (AOR 3.20) and Missouri U. S. A (AOR 3.20). There are plausible but need to be proven explanations that birth defects may result PTB. Among these, certain birth 
defects associated with abnormal connective tissue may increase the risk of preterm delivery, PTB and birth defects may have shared a common risk factors, and even not observed in our case, prenatal diagnosis of birth defect may lead to deliberately planned delivery through induction or cesarean section before 37 weeks of gestation $(29,30)$. As stated by the study done in Ethiopia, this might be due to the interaction of environmental and genetic risk factors that contribute to PTB(15). As observed in this study, it may be due to that most mothers with birth defect newborns had no iron/folic acid supplementation during current pregnancy.

Lack of iron/folic acid supplementation during current pregnancy also another significant factor for PTB. It increases the chance of PTB by four folds. A study from Kenya also showed $80 \%$ protective effect of prescribed iron/folic acid during pregnancy with PTB (AOR OR $=0.20)$. Another study from Sudan reported that folic acid level was associated with a lower risk of PTB $(O R=0.64)$ and there was a significant positive correlation between gestational age and folic acid level. This finding also supported by findings from China. The reason, in part, may be due to iron/folic acid supplementation prevents anemia and its effect. However, this finding contradicts the report from Bangladesh that iron/folic acid supplementation was a risk factor for PTB. We cannot definitively elucidate the disagreements between the studies in the association between maternal iron/folic acid supplementation and PTB, but it has been reported that the effects of hemoglobin concentration on birth outcomes could differ by ethnicity (31). It needs further interventional study.

\section{Conclusion}

The study indicated that ANC follow up fewer than four visits, PIH, no iron/folic acid supplementation during current pregnancy, being anemic and birth defect were significantly associated determinants of preterm birth.

Health education and awareness creation about the benefit of adequate ANC follow, iron/folic acid consumption, and early prevention of pregnancy-related complications are very important during pregnancy. It is also advisable to prevent and treat maternal anemia during pregnancy.

Strengthening patient tracing mechanism through phone call or home visit when mothers interrupt their follow up is advisable. Monitoring and evaluation of the effectiveness of maternal and child health/MCH services is also better.

\section{Abbreviations}

ANC: antenatal care; AOR: adjusted odd ratio; Cl: confidence interval; COR: crudes odd ratio; EDHS: Ethiopian demographic and heath survey ; HIV/ADIS: Human Immunodeficiency virus/ Acquired immunodeficiency syndrome; IQR: interquartile range ; LBW: low birth weight; LMP: last menstrual period; LNMP: Last normal menstrual period; MSc: Master of science; MUAC: mid upper arm circumference; PIH: pregnancy induced hypertension; PROM: premature rupture of membrane; PTB: preterm birth; SPSS: Statistical Package for Social Sciences; UTI: urinary tract infection; VIF: variance inflation factor 


\section{Declarations}

\section{Ethics approval and consent to participate}

The Ethical clearance was obtained from the Ethical Review Committee of School of Nursing on behalf of University of Gondar Ethical Review Board. A permission letter was also obtained from the study Hospitals' quality control office. After explanation had given about the purpose of the study, written consent was obtained from parent or legal guardian of the children. For parents or legal guardian who could not read and write, a thumbprint was used in place of the participant's signature. Anonymous questioner was used to protect the identity and confidentiality of the information obtained. Besides, once the information was gathered from the patient, it was put under a locked cupboard and in a password protected computer.

Consent to publish: Not applicable.

Availability of data and material: all relevant data are available in the main document.

\section{Competing interest}

The authors declared that we have no any competing interests.

\section{Funding}

The study was funded by Amhara Regional State Health Bureau but the funder has no role on publication.

\section{Authors' contribution}

MS wrote the proposal, participated in data collection, analyzed the data and drafted the manuscript. NBT and HSM approved the proposal with revisions, participated in data collection, data analysis and revised subsequent drafts of the manuscript. All authors read and approved the final manuscript.

\section{Acknowledgments}

We would like to thank study participants, data collectors, and supervisors for their willingness, cooperation, and commitment during data collection.

\section{References}

1. Dbstet A. WHO: recommended definitions, terminology and format for statistical tables related to the perinatal period and use of a new certificate for cause of perinatal deaths. Acta Obstet Gynecol Scand. 1977;56(3):247-53. Acta Obstet Gynecol Scand. 1977;56(3):247-53.

2. Chawanpaiboon S, Vogel JP, Moller A-B, Lumbiganon P, Petzold M, Hogan D, et al. Global, regional, and national estimates of levels of preterm birth in 2014: a systematic review and modelling analysis. Lancet Glob Health [Internet]. 2018 Oct 30 [cited 2018 Nov 11];0(0). 
3. Lawn JE, Davidge R, Paul VK, Xylander S von, de Graft Johnson J, Costello A, et al. Born Too Soon: Care for the preterm baby. Reprod Health. 2013 Nov 15;10(Suppl 1):S5.

4. March of Dimes, PMNCH, Save the Children, WHO. Born Too Soon: The Global Action Report on Preterm Birth. Eds CP Howson, MV Kinney, JE Lawn. World Health Organization. Geneva, 2012. 2012;

5. Gebreslasie K. Preterm Birth and Associated Factors among Mothers Who Gave Birth in Gondar Town Health Institutions. Adv Nurs. 2016;

6. Bekele I*, Demeke T and Dugna K. Prevalence of Preterm Birth and its Associated Factors among Mothers Delivered in Jimma University Specialized Teaching and Referral Hospital, Jimma Zone, Oromia Regional State, South West Ethiopia. J Womens Health Care. 2017

7. Li Liu, Shefali Oza, Dan Hogan, Yue Chu, Jamie Perin, Jun Zhu, Joy E Lawn, Simon Cousens, Colin Mathers, Robert E Black. Global, regional, and national causes of under-5 mortality in 2000-15: an updated systematic analysis with implications for the Sustainable Development Goals. 2016 Nov 10;

8. You D, Hug L, Ejdemyr S, Beise J. Levels and trends in child mortality. Estimates developed by the UN Inter-agency Group for Child Mortality Estimation (IGME). Report 2015.

9. Lawn JE, Kinney M. Lawn JE, Kinney M. Preterm birth: now the leading cause of child death worldwide. American Association for the Advancement of Science; 2014. Sci Transl Med. 2014 Nov 19;6(263):263ed21-263ed21.

10. Pike USNL of M 8600 R, Usa. WHO Recommendations on Interventions to Improve Preterm Birth Outcomes. [Internet]. World Health Organization; 2015 [cited 2018 Nov 12].

11. WHO, UNICEF Mathers, C. Global strategy for women's, children's and adolescents' health (2016-2030) [Internet]. WHO. [cited 2018 Nov 15].

12. Lawn JE, Kinney MV, Belizan JM, Mason EM, McDougall L, Larson J, et al. Born Too Soon: Accelerating actions for prevention and care of 15 million newborns born too soon. Reprod Health. 2013 Nov 15;10(Suppl 1):S6.

13. Oftedal A-M, Busterud K, Irgens LM, Haug K, Rasmussen S. Socio-economic risk factors for preterm birth in Norway 1999-2009: Scand J Public Health [Internet]. 2016 Jun 15 [cited 2018 Nov 15];

14. Mohammed Abaraya1 Sheka Shemsi Seid2 Shemsedin Amme Ibro2. Determinants of preterm birth at Jimma University Medical Center, southwest Ethiopia [Internet]. ResearchGate. [cited 2018 Nov 15].

15. Girmay Teklay1* , Tsega Teshale2, Hagos Tasew1, Teklewoini Mariye1, Hagos Berihu1 and Teklay Zeru1. Risk factors of preterm birth among mothers who gave birth in public hospitals of central zone, Tigray, Ethiopia: unmatched case-control study. BMC. 2018 Aug;

16. Menon R. Preterm birth: a global burden on maternal and child health. Pathog Glob Health. 2012 Jul;106(3):139-40.

17. Ververs M, Antierens A, Sackl A, Staderini N, Captier V. Which Anthropometric Indicators Identify a Pregnant Woman as Acutely Malnourished and Predict Adverse Birth Outcomes in the Humanitarian Context? PLoS Curr [Internet]. 2013 Jun 7 [cited 2018 Dec 13];5.

18. Alice M. Tang, Mei Chung, Kimberly Dong, Norma Terrin, Andrew Edmonds, Nega Assefa, Terusha Chetty, Roshan Ramlal, Parul Christian, Keith West, Naveed Janjua, Christine Wanke, Megan Deitchler, 
Zeina Maalouf-Manasseh. FANTA Project III. USAID.Determining a Global Mid-Upper Arm Circumference Cutoff to Assess Malnutrition in Pregnant Women. 2016;

19. Præstegaard C, Kesmodel PS, Kesmodel US. Is TWEAK a valid screening questionnaire to identify alcohol risk drinkers among pregnant women in Denmark? Acta Obstet Gynecol Scand. 2018;97(4):483-90.

20. US Department of Health Human Services. Dietary guidelines for Americans 2015-2020: Skyhorse Publishing Inc.; 2017. :144.

21. Tigist B, Abdela A, Kahsay ZG*. Preterm Birth and Associated Factors among Mothers Who gave Birth in Debremarkos Town Health Institutions, 2013 Institutional Based Cross Sectional Study. 2015;

22. Dorka W. Abiy S. Goitom G.: Association of maternal anthropometrics with preterm birth and determine other risk factors for preterm birth among mothers who delivered in Gandhi Memorial Hospital and Tikur Anbessa Hospital, 2016/17.

23. CSA. Ethiopia Demographic and Health Survey 2016. Addis Ababa, Ethiopia, and Rockville, Maryland, USA: CSA and ICF. 2017;

24. Wagura P, Wasunna A, Laving A, Wamalwa D, Ng'ang'a P. Prevalence and factors associated with preterm birth at kenyatta national hospital. BMC Pregnancy Childbirth [Internet].

25. Butali A, Ezeaka C, Ekhaguere O, Weathers N, Ladd J, Fajolu I, et al. Characteristics and risk factors of preterm births in a tertiary center in Lagos, Nigeria. Pan Afr Med J [Internet]. 2016

26. Alijahan R, Hazrati S, Mirzarahimi M, Pourfarzi F, Ahmadi Hadi P. Prevalence and risk factors associated with preterm birth in Ardabil, Iran. Iran J Reprod Med. 2014 Jan;12(1):47-56.

27. Zhang Q, Ananth CV, Li Z, Smulian JC. Maternal anaemia and preterm birth: a prospective cohort study. Int J Epidemiol. 2009 Oct 1;38(5):1380-9.

28. Allen LH. Biological mechanisms that might underlie iron's effects on fetal growth and preterm birth. J Nutr. 2001;131(2S-2):581S-589S.

29. Dolan SM, Gross SJ, Merkatz IR, Faber V, Sullivan LM, Malone FD, et al. The Contribution of Birth Defects to Preterm Birth and Low Birth Weight: Obstet Gynecol. 2007 Aug;110(2, Part 1):318-24.

30. Honein MA, Kirby RS, Meyer RE, Xing J, Skerrette NI, Yuskiv N, et al. The Association Between Major Birth Defects and Preterm Birth. Matern Child Health J. 2009 Mar;13(2):164-75.

31. Yi S-W, Han Y-J, Ohrr H. Anemia before pregnancy and risk of preterm birth, low birth weight and smallfor-gestational-age birth in Korean women. Eur J Clin Nutr. 2013 Apr;67(4):337-42. 